\title{
An update on breast cancer biomarkers
}

Volume 5 Issue 2 - 2017

\begin{abstract}
Breast cancer is the most common cancer that affects millions of people worldwide. Delayed diagnosis of these cancers has increased the morbidity and mortality. However, the vast research on cancer biomarkers during recent years provide a powerful and dynamic approach to understand the wide spectrum of cancers with applications in prognosis, diagnosis, screening, randomized clinical trials, and observational and analytic epidemiology. Cancer biomarkers have tremendously increased the efficacy of treatment and efficacy of detection. We have comprehensively summarized various biomarkers of breast cancer and highlighted recent research.
\end{abstract}

Keywords: biomarkers, breast cancer, VEGF, diagnosis

\author{
Braira Wahid,' Khansa Wahid, ${ }^{2}$ Sohaib Bin \\ Wahid $^{1,3}$ \\ 'Centre for Applied Molecular Biology, University of the Punjab, \\ Pakistan \\ 'Lahore College for Women University, Pakistan \\ ${ }^{3}$ Lahore Garrison University, Defence Campus Lahore, Pakistan
}

Correspondence: Braira Wahid, Centre for Applied Molecular Biology, 87-West Canal Bank Road Thokar Niaz Baig, University of the Punjab, Lahore, Pakistan,, Tel 92-42-5293।4I, Fax 92-425293149, Email brairawahid@gmail.com

Received: February 01, 2017 | Published: February 16, 2017

\begin{abstract}
Abbreviations: BC, breast cancer; EGFR, epidermal growth factor receptor; VEGF, vascular endothelial growth factor; NSE, neuron-specific enolase; $\mathrm{MBC}$, metastatic breast cancer; AA, abiraterone acetate
\end{abstract}

\section{Introduction}

Breast cancer is the leading cause of death worldwide. In 2013, Centre for Disease Prevention and Control documented that 230,815 women were diagnosed with breast cancer in United States of which 40,860 women were died. ${ }^{1}$ Delayed diagnosis or limitations of mammography lead to poor prognosis of breast cancer. Screen-film mammography (SFM) that is being used for diagnosis of breast cancer gives false positive results, only observed in aged women, and is less sensitive in women with dense breast tissue. There is dire need to overcome these limitations to improve the detection of breast cancer. Development of resistance to chemotherapeutics is another factor contributing towards increased mortality. It is crucial to develop novel biomarkers with potential to confirm diagnosis and prognosis and to predict patient's treatment choice and drug resistance development. ${ }^{2}$ This review article focuses promising biomarkers for diagnosis, prognosis, and prediction of which therapies will be efficacious for breast cancer.

\section{Circulating DNA}

Circulating DNA is ideal and non-invasive biomarker isolated from body fluids especially human serum and plasma. Small amount of free DNA circulate in both diseased and healthy human serum or plasma however, an increased plasma/serum concentration of DNA is observed in cancer patients because of higher cellular turnover in cancer cells..$^{3-5}$

Next generation sequencing of somatic tumor specific mutations such as TP53/ PIK3CA and PI3Kgene mutations in circulating DNA has successfully demonstrated that tracking tumor-specific circulating free DNA may provide valuable, accurate and feasible tool for monitoring disease and therapy in breast cancer patients. ${ }^{6,7}$ Genomic aberration specifically mutations have been detected in the hotspot regions of exon 9 and 20 of the PIK3CA in single circulating tumor cells of patients suffering from HER2-negative metastatic breast cancer. $^{8}$

Based on candidate gene approach, large number of breast- cancer related methylation biomarkers have been discovered. ${ }^{9}$ As such, regions identified lie in the promoters of cancer related gene such as $A P C,{ }^{10} R A S S F 1 A,{ }^{11}$ and SOX $17 .{ }^{12}$ Cohort-study revealed that methylated CST6 differentiated breast cancer samples from control samples. The study was based on two separate cohorts of breast-cancer patients including two separate cohorts of breast cancer patients, with methylation found in 49/123 patients (39.8\%) in one cohort, and $14 / 73$ patients $(19.2 \%)$ in other cohort, while none of the 37 healthy individuals showed methylation. ${ }^{13}$ Another retrospective study, based on 30 healthy controls and 36 early stage breast cancer patients showed that use of 8-gene biomarker panel (that included CST6) provided highest sensitivity and specificity by correctly identifying $90 \%$ of cancer negative samples and $91.7 \%$ of cancer samples. Significant level of CST6 methylation in the control samples was also observed in this study. ${ }^{10}$

Likewise, another study evaluated the ability of circulating DNA to identify the tumors in 640 patients with diverse cancer types. The results exhibited the presence of circulating DNA in $>75 \%$ patients with advanced head and neck cancers, melanoma, gastroesophageal, hepatocellular, breast, ovarian, pancreatic, colorectal, and bladder and in $<50 \%$ of primary prostate, renal, brain, and thyroid cancers. In patients with localized tumors, circulating DNA was detected in $48 \%, 50 \%, 57 \%$, and $73 \%$ of patients with pancreatic cancer, breast adenocarcinoma, gastroesophageal cancer, and colorectal cancer, respectively. ${ }^{14}$ Another prospective study included 52 women of which 30 had genomic alterations in tumor samples that were patient specific and tumor specific. Of three blood-born biomarkers assessed in this study circulating tumor DNA was found suitable for monitoring tumor cells. ${ }^{15}$

Baseline serum levels of circulating cell-free DNA (ALU247, ALU115) can predict disease prognosis because of higher concentration observed in cancer patients than in healthy controls. ${ }^{16}$ It has been recently been reported that serum cell-free DNA (cfDNA) mutations in CREBBP, SMAD4, AKAP9, PIK3CA, and TP53 act as biomarkersbreast cancer patients receiving tamoxifen. ${ }^{17}$

\section{Estrogen receptor}

Expression of estrogen receptor acts as biomarker of breast cancer, because it provides the index for sensitivity to endocrine treatment. Estrogen expression increased with age in breast cancer patients. ${ }^{18}$ 
Likewise, ER (estrogen receptor) level in TDLU-PT (terminal duct lobular units- proximal to tumor) and TDLU-DT (terminal duct lobular units-distal to tumor) was higher in postmenopausal women. ${ }^{19}$ A recent study has demonstrated that neo-tanshinlactone decreased steady state expression level of ESR1 mRNA in ER+ breast cancer cells. Analysis of ER mRNA levels and protein levels of target genes of transcription factor especially GREB1, CCND1, TFF1, BRCA1, ESR2, ABCA3, and SERPINB9 further confirms the aforementioned finding. ${ }^{20}$ ER negative and PR positive breast cancer is not a reproducible subtype. The expression of progesterone receptor is not associated with prognosis of estrogen receptor negative breast cancer. ${ }^{21} \mathrm{~K} 303 \mathrm{R}$ ER mutation has been associated with breast cancer and benign breast hyperplasia. ${ }^{22}$ An abiraterone acetate (AA) pharmacodynamic effect have been found to increase progesterone and decrease serum androgen and estrogen levels. Dual expression of ER and AR in circulating tumor cells and newly obtained formalinfixed paraffin-embedded tissues may predict AA sensitivity. ${ }^{23}$ Tumor apparent diffusion coefficient is being investigated as imaging biomarker to predict tumor aggressiveness in patients with estrogenreceptor-positive breast cancer. ${ }^{24} \mathrm{ER}-\alpha$ is expressed in about $70 \%$ of breast cancers therefore, it ER may serve as predictor of response to endocrine therapy and confirm resistance to certain endocrine therapies. ${ }^{25}$

\section{Progesterone receptor (PgR)}

The expression of progesterone receptor depends upon estrogen receptor. Less than $1 \%$ of tumors in all breast cancer cases express progesterone receptor but not estrogen receptor. Such reanalysis of the ER status of such tumors is necessary to eliminate false ER negativity. ${ }^{26}$ Absence of PR expression acts as powerful and independent biomarker for prognosis in ER-positive breast cancer patients receiving endocrine therapy. ${ }^{27}$ Initial studies suggested poor prognosis in ER-positive/PgR-negative breast compared to PgR-positive tumors. ${ }^{28}$ ATAC (Arimidex, Tamoxifen, Alone or in Combination) trialist group demonstrated that patients with $\mathrm{PgR}$ negative breast cancer patients gain more benefit from anastrozole than tamoxifen compared with PgR-positive patients. ${ }^{29}$ These results were not confirmed in centrally analyzed material from $\mathrm{PgR}$ and/or 1856 ER expressing patients. The BIG 1-98 trial revealed that PgR status had not varied the efficacy of letrozole over tamoxifen. ${ }^{26}$ All these adjuvant therapies exhibited the association of PgR expression level and prognosis of endocrine therapy. Evaluation of degree of correlation between immunohistochemical and immunocytochemical determination of PR and ER in breast cancer showed that both PR and ER were negative in nine cases but positive in four cancer (PR in 1 and ER in 3). All these evidences support the use of ER and PR in prognosis of breast cancer. ${ }^{30}$

\section{Androgen receptor}

The androgen receptor is well-studied in context of male physiology because of its proven clinical target in prostate cancer. Basal cytokeratins and androgen receptors provide powerful prognostic information in triple-negative breast cancer. ${ }^{31}$

Several studies show that association of breast cancer survival with AR status depends upon ER expression thus causing ameliorated survival in ER+ tumors. The expression level of AR was found to be considerably higher in endocrine responsive tumors. Multivariate analysis of disease free survival demonstrated the importance of AR for prognosis and survival outcome. The results of the study exhibited that $90 \%$ of ER-positive patients received chemo-endocrine therapy and endocrine therapy thus, suggesting the prediction of systematic treatments. ${ }^{32}$ Whereas, in ER negative and triple negative breast cancer subset no prognosis or poor prognosis impact of ER and AR negative tumor patients was observed compared to ER-positive tumor patients. AR was found to be more frequently expressed than ER and PR in another study that recruited 980 Chinese patients with invasive breast carcinoma. However, the expression of AR was closely associated with PR and ER with higher level observed in ER+ subtypes than in ER- subtypes. It was related to more favorable pathological features such as marker of proliferation in breast cancer, lower Ki-67 expression and small tumor size. ${ }^{33}$

Another retrospective study based on 287 females diagnosed with TNBC (triple negative breast cancer) showed that AR were present at rate of $25.8 \%$ in TNBC lower than in ER-positive breast cancers. AR positivity was associated with longer overall survival and disease free survival whereas, poor prognosis was noticed in ARnegative patients. ${ }^{34}$ In the prospective GeparTrio phase-III trial, tissue microarray of 673 core biopsies from primary breast cancer patients were treated with neoadjuvant cyclophosphamide/ doxorubicin/ docetaxel chemotherapy followed by immunohistochemistry to predict the effect of AR on pathological response and its role as prognostic marker specifically in patients not attaining pathological complete response and triple negative breast cancer. ${ }^{35}$ The response of breast cancer to traditional endocrine therapy is influenced by AR to ER ratio which makes them a promising biomarker of breast cancer. ${ }^{36}$

\section{Human epidermal growth factor receptor-2 (HER2)}

The HER2 (ERBB2) oncogene encodes a transmembrane tyrosine kinase receptor. Over-expression of HER 2 in $10-25 \%$ of breast cancers makes it an effective therapeutic treatment target and biomarker for breast cancer and is a validated adverse prognostic factor with a mean relative risk for overall survival of 2.74. ${ }^{37,38}$ Accumulating evidence suggest an extraordinary therapy in HER2-positive breast because of poor prognosis observed in HER2-positive breast cancer than HER2negative breast cancer. ${ }^{39}$

A recent study investigated the predictive value of CTC (Circulating Tumor Cell) HER2 expression and prognostic significance of CTC enumeration for efficient anti-HER2 therapy in HER2-positive metastatic breast cancer (MBC) patients. The results provided valuable predictive and prognostic information to optimize therapies in HER-2 positive MBC patients..$^{40}$ Researchers found direct tumour-promoting interactions of UPAR with UPA and the insulinlike growth factor receptor 1 (IGF1R) in triple negative breast cancer cells thus, suggesting the role of UPAR as biomarker and therapeutic target. ${ }^{41}$ Novel interacting partners of uPAR i.e, Cyr61 and YB-1 may also act as therapeutic targets because of their reported elevation in the malignancy of triple-negative breast cancer. ${ }^{42}$ It has been reported that $\mathrm{BC}$ cause brain metastasis but there is no biomarker yet available that predicts brain metastasis. Neuron-specific enolase (NSE) and serum $\mathrm{S} 100 ß$ protein are elevated in brain damages induced by brain metastasis. Matrix metalloproteinase 9 (MMP-9), NSE, and HER2 have a particular tropism for central nervous system (CNS) these three biomarkers are correlated with $\mathrm{BM}$ in metastatic $\mathrm{BC}$ patients. ${ }^{43}$ In vivo, CDK4/6 inhibitors have found to delay recurrence of tumor in transgenic model of HER-2 positive breast cancer because CDK4/6 inhibitors sensitize patient-derived xenograft tumors to HER2targeted therapies. ${ }^{44}$

\section{MicroRNAs (miRNAs)}

mircroRNAs act as tumor suppressor genes or oncogenes because dysregulation of miRNAs plays an important role in the initiation and progression of different human cancers, including breast cancer 
(BC). Several different miRNAs act as biomarkers that predict therapeutic outcomes miR-30c, miR-339-5p, miR-187, prognostic biomarkers $m i R-148 a, m i R-33 a$, and diagnostic biomarkers $m i R$ 9, $m i R-10 b$, and $m i R-17-5 p$. All these miRNAs play diverse role in controlling metastasis, invasion, proliferation, apoptosis, genomic instability, and resting death. Other important miRNAs of breast cancer that circulate in body fluids are $m i R-155, m i R-210 .{ }^{45,46}$

\section{Vascular endothelial growth factor (VEGF)}

The gene expression of VEGF is controlled by several different stimuli such as growth factors, nitric oxide, hypoxia, HER-2, tumor suppressor genes, and oncogenes. ${ }^{47} \mathrm{VEGF}$ regulates neovascularization in the tumor by elevating expression of XIAP, survivin, and Bcl2 that are anti-apoptotic proteins. ${ }^{48}$ The expression of VEGF is increased in invasive breast cancer and ductal carcinoma in citu therefore, it can be used to predict the prognosis of breast cancer. ${ }^{49}$

Recent studies confirmed the correlation between VEGF level and larger tumour size, grade III tumours, and negative hormone status. Level of VEGF decreases with chemotherapy and poor survival is observed. In triple negative breast cancer higher level of VEGF is related to original survival, disease free survival, and distant disease-free survival. Tumor size, grade, and metastatic sites are also influenced by VEGF levels. Despite of therapy, disease progresses in the patients with high VEGF levels. Significantly lower progression free survival is noticed in patients with high VEGF level compared to those with lower levels. ${ }^{50}$ In triple negative breast cancer patients bevacizumab targets VEGF. ${ }^{51}$ All this data suggest its promising role as biomarker.

\section{Epidermal growth factor receptor (EGFR)}

Overexpression of EGFR in all types of breast cancers (specifically in triple-negative breast cancer and inflammatory breast cancer) is associated with poor differentiation, poor clinical outcomes, and large tumor size. Several therapies including apatinib, cetuximab, gefitinib have been shown to target EGFR. Mutations in EGFR genes cause the overexpression of EGFR. A study reported EGFR mutation in 11 of 24 hereditary breast carcinomas and 7 of 48 sporadic breast carcinomas. ${ }^{52}$ EGFR expression level or gene mutation status may act as predictive biomarker. ${ }^{53}$ Phosphorylation of EGFR may act as biomarker of response to time-staggered inhibition in EGFR driven tumor types such as lung cancer and triple negative breast cancer. ${ }^{54,55} \mathrm{~A}$ recent study revealed that immunoexpression of biomarkers like CK5/6 and EGFR predict the survival of triple negative breast cancer patients. ${ }^{56}$ Likewise, recent study revealed that combined measurement of three non-intuitive proteins EGFR, ERBB3, and CDKN1B determines responsiveness to drugs targeting their constituents and cellular dependence on PI3K vs. MAPK signaling pathways. ${ }^{57}$

\section{Other genetic biomarkers}

Over the last few years, retrospective and clinical data suggests the use of different genes as biomarkers of response to therapy as well as diagnosis. Level of CA15-3 and CEA in serum predict prognosis in breast cancer. A study showed an elevation in serum levels of CA15$3 \mathrm{~A}$ and CEA in $60(13.9 \%)$ and $47(10.9 \%)$, respectively. ${ }^{58}$ Alabama Breast Cancer Project has investigated the independent prognostic significance of p53 and c-erbB-2 expression in breast cancer. ${ }^{59}$ Preclinical studies have also confirmed the chemosensitive nature of BRCA-1 mutations in breast cancer patients therefore, it can be used as predictive marker of response to different types of chemotherapy agents. ${ }^{60}$ The microarray data analysis of 1809 patients to assess effect of 22,277 genes on breast cancer prognosis revealed the high prognostic power of TOP2A, TOP2B, MKI67, CCND3, CCND2, CDKN1A, CCNE2, and TK2. ${ }^{61}$ Mammalian target of rapamycin, TK, Cox-2, heat shock protein 90, PARP, Ki67, and TOP-2A are important biomarkers of triple negative breast cancer. ${ }^{62}$

\section{Emerging biomarkers of future}

Epigenetic changes especially DNA methylation has shown promise as biomarker of prognosis and diagnosis. ${ }^{63,64}$ Oligonucleotide microarrays and cDNA are being applied to study breast cancer to devise predictive "signatures" in the setting of neoadjuvant chemotherapy setting. ${ }^{65}$

The activity of PI3K/PTEN pathway in ER-negative and basallike breast cancer supports the future clinical evaluation of combining PI3K with EGFR pathway inhibitors for the treatment of breast like breast cancer. ${ }^{66}$

In breast cancer, PIK3CA genotype is influenced by LRG1 mRNA expression and aromatase inhibitor therapy. A recent study included 9 patients who developed metastatsis, 15 patients with favourable outcome, and 24 patients with invasive ductal breast cancer were kept under observation for 5 years. Set of 58 differentially expressed genes $(p \leq 0.01)$ between the two groups were identified in global gene expression profiles that was generated by using tumor samples from all patients. RT-qPCR confirmed that GTSE1, B3GNT7, $P P M 1 D, T N K S 2$, and $P H B$ were upregulated in patients with poor outcomes. Oligoarray data revealed reduced BCL2-associated agonist of cell death (BAD) mRNA expression and considerable BAD protein expression in metastatic cases and $1276 \mathrm{BC}$ tissue samples respectively. These findings form the basis of development of novel prognostic markers that can differentiate breast carcinomas with favorable outcomes and breast carcinoma with metastatic potential. ${ }^{67}$

Recent evaluation of 15 biomarkers revealed that PIK3CA, CYP2D6, RARA, TIMP-1, STAT3, and Lin-28 could predict tumor drug response furthermore, combination of h-MAM, snail, osteopontin, twist, FGFR, zeb-1, PTEN and sirtuins, uPA, PAI and TF have the potential to confirm the diagnosis and prognosis of breast cancer. ${ }^{2}$

The study directed at validation of Nottingham Prognostic Index Plus (NPI+) in an independent series of breast cancer (BC) assessed 885 primary early stage $\mathrm{BC}$ cases for 10 biomarkers (PgR, ER, cytokeratin (CK) 5/6, CK7/8, HER2, HER3, HER4, EGFR, Mucin 1 , and $\mathrm{p} 53$ that confirmed the prognostic value of these biomarkers. ${ }^{68}$ Long noncoding RNAs (lncRNAs), cyclin E, cyclin D1, Ki67, and ER- $\beta$ are also emerging as prognostic biomarker in aggressive breast cancer. ${ }^{69-70}$

\section{Conclusion}

Despite of vast research conducted on breast cancer biomarkers during last decade, there are certain gaps that are still to be filled to bring newly acquired knowledge into clinical practice. This review discussed prognostic and diagnostic potential of several biomarkers however, there is a need to validate the expression of these potential biomarkers in large patient cohorts. The established clinicopathologic markers, such as ER, HER2, miRNA, p53, have clearly defined clinical applicability.

\section{Acknowledgements}

None.

\section{Conflicts of interest}

Author declares there are no conflicts of interest. 


\section{Funding}

None.

\section{References}

1. CDC. Breast Cancer Statistics. Centre for Disease Prevention and Control, USA. 2013.

2. dos Anjos Pultz B, da Luz FA, de Faria PR, et al. Far Beyond the Usual Biomarkers in Breast Cancer: a review. J Cancer. 2014;5(7):559-571.

3. Gahan PB, Swaminathan R. Circulating nucleic acids in plasma and serum. Recent developments. Ann N Y Acad Sci. 2008;1137:1-6.

4. Dachuan Jin, Suqing Xie, Zhicheng Mo, et al.) Circulating DNAimportant biomarker of cancer. Journal of Molecular Biomarkers \& Diagnosis. 2012.

5. Kohler C, Barekati Z, Radpour R, et al. Cell-free DNA in the circulation as a potential cancer biomarker. Anticancer res. 2011;31(8):2623-2628.

6. Nakauchi, Kagara N, Shimazu K, et al. Detection of TP53/PIK3CA Mutations in Cell-Free Plasma DNA From Metastatic Breast Cancer Patients Using Next Generation Sequencing. Clin Breast Cancer. 2016;16(5):418-423.

7. Li Z, Guo X, Tang L, et al. Methylation analysis of plasma cell-free DNA for breast cancer early detection using bisulfite next-generation sequencing. Tumor Biol . 2016;37(10):13111-13119.

8. Gasch C, Oldopp T, Mauermann O, et al. Frequent detection of PIK3CA mutations in single circulating tumor cells of patients suffering from HER2-negative metastatic breast cancer. Mol Oncol . 2016;10(8):1330-1343.

9. Guerrero-Preston R, Hadar T, Ostrow KL, et al. Differential promoter methylation of kinesin family member 1a in plasma is associated with breast cancer and DNA repair capacity. Oncol Rep.2014;32(2):505-512.

10. Radpour R, Barekati Z, Kohler C, et al. Hypermethylation of tumor suppressor genes involved in critical regulatory pathways for developing a blood-based test in breast cancer. PLoS One. 2011;6(1):e16080.

11. Hoque MO, Feng Q, Toure P, et al. Detection of aberrant methylation of four genes in plasma DNA for the detection of breast cancer. $J$ Clin Oncol. 2006;24(26):4262-4269.

12. Chimonidou M, Strati A, Malamos N, et al. SOX17 promoter methylation in circulating tumor cells and matched cell-free DNA isolated from plasma of patients with breast cancer. Clin Chem. 2013;59(1):270-279.

13. Li M, Chen WD, Papadopoulos N, et al. Sensitive digital quantification of DNA methylation in clinical samples. Nat Biotechnol. 2009;27(9):858-863.

14. Bettegowda C, Sausen M, Leary RJ, et al. Detection of circulating tumor DNA in early-and late-stage human malignancies. Sci transl med. 2014;6(224):224ra24

15. De Mattos-Arruda L, Cortes J, Santarpia L, et al. Circulating tumour cells and cell-free DNA as tools for managing breast cancer. Nat Rev Clin Oncol . 2013;10(7):377-389.

16. Iqbal S, Vishnubhatla S, Raina V, et al. Circulating cell-free DNA and its integrity as a prognostic marker for breast cancer. Springerplus. $2015 ; 4: 265$

17. Jansen MP, Martens JW, Helmijr JC, et al. Cell-free DNA mutations as biomarkers in breast cancer patients receiving tamoxifen. Oncotarget . 2016;7(28):43412-43418.

18. Droog M, Mensink M, Zwart W. The Estrogen Receptor $\alpha$ cistrome beyond breast cancer. Mol Endocrinol. 2016;30(10):1046-1058.

19. Yang XR, Figueroa JD, Hewitt SM, et al. Estrogen receptor and progesterone receptor expression in normal terminal duct lobular units surrounding invasive breast cancer. Breast Cancer Res Treat. 2013;137(3):837-847

20. Lin W, Huang J, Liao X, et al. Neo-tanshinlactone selectively inhibits the proliferation of estrogen receptor positive breast cancer cells through transcriptional down-regulation of estrogen receptor alpha. Pharmacol Res. 2016;111:849-858.

21. Hefti MM, Hu R, Knoblauch NW, et al. Estrogen receptor negative/ progesterone receptor positive breast cancer is not a reproducible subtype. Breast Cancer Res. 2013;15(4):R68.

22. Herynk MH, SA Fuqua. Estrogen receptor mutations in human disease. Endocr rev. 2004;25(6):869-898.

23. Li W, Hayes D, Campone M, et al. Biomarker Associations with Efficacy of Abiraterone Acetate and Exemestane in Postmenopausal Patients with Estrogen Receptor-Positive Metastatic Breast Cancer. Clin Cancer Res. 2016;22(24):6002-6009.

24. Shin HJ, Kim SH, Lee HJ, et al. Tumor apparent diffusion coefficient as an imaging biomarker to predict tumor aggressiveness in patients with estrogen-receptor-positive breast cancer. NMR Biomed. 2016;29(8):1070-1078.

25. Lauring J, Wolff AC. Evolving Role of the Estrogen Receptor as a Predictive Biomarker: ESR1 Mutational Status and Endocrine Resistance in Breast Cancer, American Society of Clinical Oncology. 2016.

26. Viale G, Regan MM, Maiorano E, et al. Prognostic and predictive value of centrally reviewed expression of estrogen and progesterone receptors in a randomized trial comparing letrozole and tamoxifen adjuvant therapy for postmenopausal early breast cancer: BIG 1-98. J Clin Oncol. 2007;25(25):3846-3852.

27. Purdie CA, Quinlan P, Jordan LB, et al. Progesterone receptor expression is an independent prognostic variable in early breast cancer: a population-based study. Br J Cancer . 2014;110(3):565-572.

28. Hammond ME, Hayes DF, Dowsett M, et al. American Society of Clinical Oncology/College of American Pathologists guideline recommendations for immunohistochemical testing of estrogen and progesterone receptors in breast cancer. Arch Pathol Lab Med. 2010;134(7):907-922.

29. Howell A, Cuzick J, Baum M, et al. Results of the ATAC (Arimidex, Tamoxifen, Alone or in Combination) trial after completion of 5 years' adjuvant treatment for breast cancer. Lancet. 2005;365(9453):60-62.

30. Radhika K, AK Prayaga. Estrogen and progesterone hormone receptor status in breast carcinoma: Comparison of immunocytochemistry and immunohistochemistry. Indian J Cancer . 2010;47(2):148-150.

31. Rakha EA, El-Sayed ME, Green AR, et al. P Prognostic markers in triple-negative breast cancer. Cancer. 2007; 109(1):25-32.

32. Park S, Koo JS, Kim MS, et al. Androgen receptor expression is significantly associated with better outcomes in estrogen receptorpositive breast cancers. Ann Oncol. 2011;22(8):1755-1762.

33. Qi JP, Yang YL, Zhu H, et al. Expression of the androgen receptor and its correlation with molecular subtypes in 980 Chinese breast cancer patients. Breast cancer (Auckl). 2012;6:1-8.

34. He J, Peng R, Yuan Z, et al. Prognostic value of androgen receptor expression in operable triple-negative breast cancer: a retrospective analysis based on a tissue microarray. Med Oncol. 2012;29(2):406-410.

35. Loibl S, Müller BM, von Minckwitz G, et al. Androgen receptor expression in primary breast cancer and its predictive and prognostic value in patients treated with neoadjuvant chemotherapy. Breast Cancer Res Treat . 2011;130(2):477-487.

36. Cochrane DR, Bernales S, Jacobsen BM, et al. Role of the androgen receptor in breast cancer and preclinical analysis of enzalutamide. Breast Cancer Res. 2014;16(1):R7. 
37. Owens MA, BC Horten, MM Da Silva. HER2 amplification ratios by fluorescence in situ hybridization and correlation with immunohistochemistry in a cohort of 6556 breast cancer tissues. Clinical breast cancer. 2004;5(1):63-69.

38. Ross JS. Breast cancer biomarkers and HER2 testing after 10 years of anti-HER2 therapy. Drug News Perspect . 2009;22(2):93-106.

39. Telli ML, Hunt SA, Carlson RW,et al. Trastuzumab-related cardiotoxicity: calling into question the concept of reversibility. Journal of Clinical Oncology. 2007;25(23):3525-3533.

40. Liu Y, Liu Q, Wang T, et al. Circulating tumor cells in HER2-positive metastatic breast cancer patients: a valuable prognostic and predictive biomarker. BMC cancer . 2013;13:202.

41. Huber MC, Mall R, Braselmann H, et al. uPAR enhances malignant potential of triple-negative breast cancer by directly interacting with uPA and IGF1R. BMC Cancer. 2016;16(1):615

42. Huber MC, Falkenberg N, Hauck SM, et al. Cyr61 and YB-1 are novel interacting partners of UPAR and elevate the malignancy of triplenegative breast cancer. Oncotarget. 2016;7(28):44062-44075.

43. Darlix A, Lamy PJ, Lopez-Crapez E, et al. Serum NSE, MMP-9 and HER2 extracellular domain are associated with brain metastases in metastatic breast cancer patients: Predictive biomarkers for brain metastases? Int J Cancer . 2016;139(10):2299-2311.

44. Goel S, Wang Q, Watt AC, et al. Overcoming Therapeutic Resistance in HER2-Positive Breast Cancers with CDK4/6 Inhibitors. Cancer cell . 2016;29(3):255-269.

45. Bertoli G, Cava C, Castiglioni I . MicroRNAs: New Biomarkers for Diagnosis, Prognosis, Therapy Prediction and Therapeutic Tools for Breast Cancer. Theranostics. 2015;5(10):1122-1143.

46. Damavandi Z, Torkashvand S, Vasei M, et al. Aberrant Expression of Breast Development-Related MicroRNAs, miR-22, miR-132, and miR212, in Breast Tumor Tissues. J Breast Cancer. 2016;19(2):148-155.

47. Benjamin LE, E Keshet. Conditional switching of vascular endothelial growth factor (VEGF) expression in tumors: induction of endothelial cell shedding and regression of hemangioblastoma-like vessels by VEGF withdrawal. Proc Natl Acad Sci, USA. 1997;94(16):8761-8766.

48. Gerber HP, McMurtrey A, Kowalski J, et al. Vascular endothelial growth factor regulates endothelial cell survival through the phosphatidylinositol 3'-kinase/Akt signal transduction pathway. Requirement for Flk-1/KDR activation. J Biol Chem. 1998;273(46):30336-30343.

49. Chanana P, Awadesh KP, Budhi SY, et al. Significance of serum vascular endothelial growth factor and cancer antigen 15.3 in patients with triple negative breast cancer. Journal of Radiotherapy in Practice. 2014;13(1):60-67.

50. El-Arab LR, Swellam M, El Mahdy MM. Metronomic chemotherapy in metastatic breast cancer: impact on VEGF. J Egypt Natl Canc Inst. 2012;24(1):15-22.

51. Cameron D, Brown J, Dent R, et al. Adjuvant bevacizumab-containing therapy in triple-negative breast cancer (BEATRICE): primary results of a randomised, phase 3 trial. Lancet oncol . 2013;14(10):933-942.

52. Gonzalez-Angulo AM, Timms KM, Liu S, et al. Incidence and outcome of BRCA mutations in unselected patients with triple receptor-negative breast cancer. Clin Cancer Res. 2011;17(5):1082-1089.

53. Lynch TJ, Bell DW, Sordella R, et al.Activating mutations in the epidermal growth factor receptor underlying responsiveness of non-small-cell lung cancer to gefitinib. $N$ Engl J Med. 2004;350(21):2129-2139.
54. Lee MJ, Ye AS, Gardino AK, et al. Sequential application of anticancer drugs enhances cell death by rewiring apoptotic signaling networks. Cell .2012;149(4):780-794.

55. Zhang L, Fang $\mathrm{C}, \mathrm{Xu} \mathrm{X}$, et al. Androgen receptor, EGFR, and BRCA1 as biomarkers in triple-negative breast cancer: a meta-analysis. Biomed Res Int . 2015;2015:357485

56. Yue Y, Astvatsaturyan K, Cui X, et al. Stratification of Prognosis of Triple-Negative Breast Cancer Patients Using Combinatorial Biomarkers. PLoS One. 2016;11(3):e0149661.

57. Kirouac DC, Du J, Lahdenranta J, et al. HER2+ Cancer Cell Dependence on PI3K vs. MAPK Signaling Axes Is Determined by Expression of EGFR, ERBB3 and CDKN1B. PLoS Comput Biol. 2016;12(4):e1004827.

58. Shao Y, Sun X, He Y, et al. Elevated levels of serum tumor markers CEA and CA15-3 are prognostic parameters for different molecular subtypes of breast cancer. PLoS One. 2015;10(7):e0133830.

59. Beenken SW, Grizzle WE, Crowe DR, et al. Molecular biomarkers for breast cancer prognosis: coexpression of c-erbB-2 and p53. Ann Surg. $2001 ;(5): 630-638$

60. James CR, Quinn JE, Mullan PB, et al. BRCA1, a potential predictive biomarker in the treatment of breast cancer. Oncologist. 2007;12(2):142-150.

61. Györffy B, Lanczky A, Eklund AC, et al. An online survival analysis tool to rapidly assess the effect of 22,277 genes on breast cancer prognosis using microarray data of 1,809 patients. Breast cancer research and treatment. 2010;123(3):725-731.

62. Yadav BS, P Chanana, S Jhamb. Biomarkers in triple negative breast cancer: A review. World J Clin Oncol . 2010;6(6):252-263.

63. Hinestrosa MC, Dickersin K, Klein P, et al. Shaping the future of biomarker research in breast cancer to ensure clinical relevance. Nature Reviews Cancer. 2007;7(4):309-315.

64. Martens JW, Margossian AL, Schmitt M, et al. DNA methylation as a biomarker in breast cancer. Future Oncol . 2009;5(8):1245-1256.

65. Reis-Filho J, Westbury C, Pierga J. The impact of expression profiling on prognostic and predictive testing in breast cancer. J Clin Pathol. 2006;59(3):225-231.

66. She QB, Gruvberger-Saal SK, Maurer M, et al. Integrated molecular pathway analysis informs a synergistic combination therapy targeting PTEN/PI3K and EGFR pathways for basal-like breast cancer. $B M C$ cancer. 2016;16:587.

67. Canevari RA, Marchi FA, Domingues MA, et al. Identification of novel biomarkers associated with poor patient outcomes in invasive breast carcinoma. Tumor Biol . 2016;37(10):13855-13870.

68. Green AR, Soria D, Stephen J, et al. Nottingham prognostic index plus: validation of a clinical decision making tool in breast cancer in an independent series. J Pathol Clin Res. 2016;2(1):32-40.

69. Sas-Chen A, Aure MR, Leibovich L, et al. LIMT is a novel metastasis inhibiting lncRNA suppressed by EGF and downregulated in aggressive breast cancer. EMBO Mol Med. 2016;8(9):1052-1064.

70. Weigel MT, Dowsett M. Current and emerging biomarkers in breast cancer: prognosis and prediction. Endocrine-Relat Cancer. 2010;17(4):R245-R262. 